\title{
The large-scale anisotropy with the PAMELA calorimeter
}

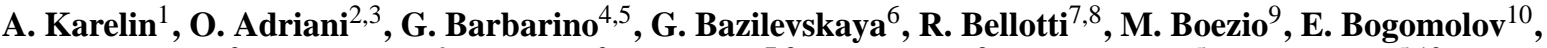 \\ M. Bongi ${ }^{3}$, V. Bonvicini ${ }^{9}$, S. Bottai ${ }^{3}$, A. Bruno ${ }^{7,8}$, F. Cafagna $^{8}$, D. Campana ${ }^{5}$, R. Carbone ${ }^{5,12}$, \\ P. Carlson ${ }^{13}$, M. Casolino ${ }^{11}$, G. Castellini ${ }^{14}$, C. De Donato ${ }^{11,12}$, C. De Santis ${ }^{11,12}$, N. De Simone ${ }^{11,12}$, \\ V. Di Felice ${ }^{11}$, V. Formato ${ }^{9}, 15$, A. Galper ${ }^{1}$, S. Koldashov ${ }^{1}$, S. Koldobskiy ${ }^{1}$, S. Krut'kov ${ }^{10}$, A. Kvashnin ${ }^{6}$, \\ A. Leonov ${ }^{1}$, V. Malakhov ${ }^{1}$, L. Marcelli ${ }^{11}$, M. Martucci ${ }^{11,17}$, A. Mayorov ${ }^{1}$, W. Menn ${ }^{16}$, M. Mergé ${ }^{11,12}$, \\ V. Mikhailov ${ }^{1}$, E. Mocchiutti ${ }^{9}$, A. Monaco ${ }^{8}$, N. Mori $^{2,3}$, R. Munini ${ }^{9,15}$, G. Osteria ${ }^{5}$, F. Palma ${ }^{11,12}$,

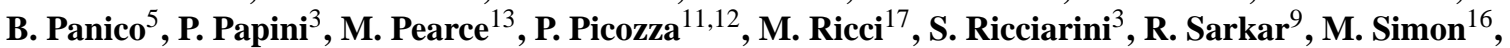 \\ V. Scotti $^{4,5}$, R. Sparvoli ${ }^{11,12}$, P. Spillantini ${ }^{2,3}$, Y. Stozhkov ${ }^{6}$, A. Vacchi ${ }^{9}$, E. Vannuccini ${ }^{3}$, G. Vasilyev $^{10}$, \\ S. Voronov ${ }^{1}$, Y. Yurkin ${ }^{1}$, G. Zampa ${ }^{9}$, and N. Zampa ${ }^{9}$ \\ ${ }^{1}$ National research nuclear university MEPhI, 115409, Moscow, Russia \\ ${ }^{2}$ University of Florence, 50019 Sesto Fiorentiono, Florence, Italy \\ ${ }^{3}$ INFN, Sezione di Florence, 50019 Sesto Fiorentiono, Florence, Italy \\ ${ }^{4}$ University of Naples "Federico II", 80126 Naples, Italy \\ ${ }^{5}$ INFN, Sezione di Naples, 80126 Naples, Italy \\ ${ }^{6}$ Lebedev Physical Institute, 119991 Moscow, Russia \\ ${ }^{7}$ University of Bari, 70126 Bari, Italy \\ ${ }^{8}$ INFN, Sezione di Bari, 70126 Bari, Italy \\ ${ }^{9}$ INFN, Sezione di Trieste, 34149 Trieste, Italy \\ ${ }^{10}$ Ioffe Physical Technical Institute, 194021 St. Petersburg, Russia \\ ${ }^{11}$ INFN, Sezione di Rome "Tor Vergata", 00133 Rome, Italy \\ ${ }^{12}$ University of Rome "Tor Vergata", 00133 Rome, Italy \\ ${ }^{13} \mathrm{KTH}$, Department of Physics, and the Oskar Klein Centre for Cosmoparticle Physics AlbaNova University \\ Centre, 10691 Stockholm, Sweden \\ ${ }^{14}$ IFAC, 50019 Sesto Fiorentino, Florence, Italy \\ ${ }^{15}$ University of Trieste, 34147 Trieste, Italy \\ ${ }^{16}$ University of Siegen, 57068 Siegen, Germany \\ ${ }^{17}$ INFN, Laboratori Nazionali di Frascati, Via Enrico Fermi 40, 00044 Frascati, Italy
}

Correspondence to: A. Karelin (karelin@ hotbox.ru)

Received: 7 May 2015 - Accepted: 24 September 2015 - Published: 2 October 2015

Abstract. The large-scale anisotropy (or the so-called star-diurnal wave) has been studied using the calorimeter of the space-born experiment PAMELA. The cosmic ray anisotropy has been obtained for the Southern and Northern hemispheres simultaneously in the equatorial coordinate system for the time period 2006-2014. The dipole amplitude and phase have been measured for energies $1-20 \mathrm{TeV} \mathrm{n}^{-1}$. 


\section{Introduction}

Despite the assumption that the arrival direction of cosmic rays should be distributed isotropically due to the different processes that they undergo during their propagation, scientists have been searching for any anisotropies since the discovery of cosmic rays in 1912. Till recent years, searches for anisotropies were conducted with ground-based experiments. Moreover, until the 1990s the obtained results were strongly affected by atmospheric conditions and imperfections on experimental equipment. A further limitation was that the experiments were carried out in a single (mostly in the Northern) hemisphere, restricting the angular range of the anisotropy measurement. The first results of largescale anisotropy measurements obtained from satellite data are presented in this paper. The dipole phase and amplitude were measured in a two-dimensional scale in an equatorial coordinate system.

\section{The PAMELA experiment}

The PAMELA magnetic spectrometer is a satellite experiment that was launched in the summer of 2006 and has been operating since then (Adriani et al., 2014). The main scientific goals of the experiment are the study of particle and antiparticle fluxes in a wide energy range. The PAMELA apparatus consist of several various detectors positioned around a magnetic spectrometer (tracker). While the tracker is able to measure the deflection of particles in the magnetic field up to energies of about $1 \mathrm{TeV}$, another PAMELA subdetector - a calorimeter, can be used to extend the measured energy range. Furthermore the calorimeter allows us to measure particle direction over a wide range of angles. The calorimeter consists of 44 silicon planes, with 96 strip detectors in each one; interleaved with 22 tungsten layers. In neighboring silicon planes, strips are orthogonal, providing topological and longitudinal information of the shower development.

\subsection{The particle selection and reconstruction of their arrival}

To measure the particle direction, the shower axis inside the calorimeter was used. The iterative procedure was used to restore the axis along the primary particle track throughout the 44 planes (Borisov et al., 2013). This procedure is a fit of the center of gravity of energy released in each plane of each view. The axis reconstruction is possible when inclination of the particle direction respect to the vertical of the calorimeter is less than $15^{\circ}$. Events for which the shower axis was reconstructed were further selected based on their total energy deposition in the calorimeter. A cut threshold was set at the level of $180000 \mathrm{mip}$ corresponding to particles with energy $1-20 \mathrm{TeV} \mathrm{nuc}^{-1}$. The obtained statistics allowed the study of the anisotropy in a one-dimensional map as a function of right ascension - RA.

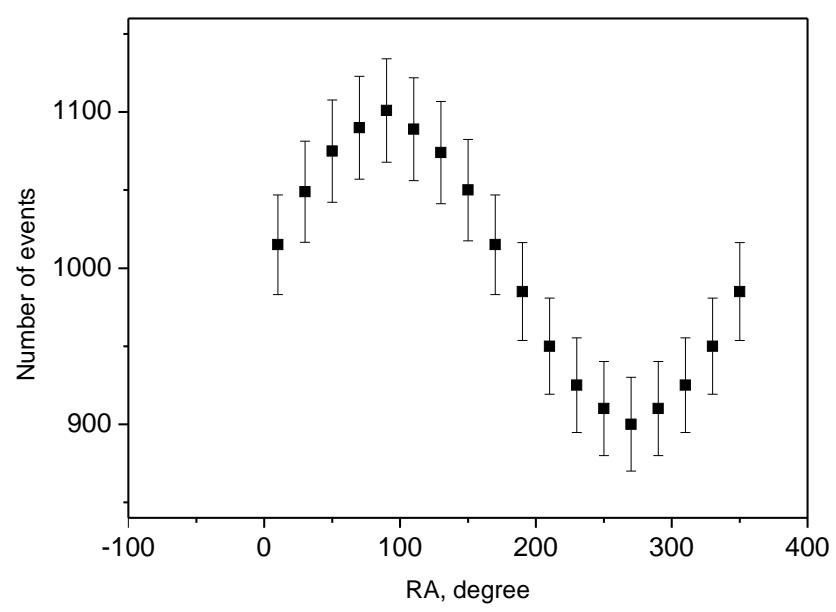

Figure 1. The simulated dipole anisotropy with an amplitude of $10 \%$.

\subsubsection{The isotropy map creation}

To create an isotropy map for comparison with the experimental one a shuffling method was used (for details see Karelin et al., 2013). The idea of this method is to randomize the reconstructed directions of events. A set of isotropic simulated events can be built by randomly coupling the times and the directions of real events in local instrument coordinates. The randomization is implemented starting with the position of a given event in the PAMELA frame and exchanging it with the direction of another event, which was selected randomly from the data set with a uniform probability. Since this method was designed mostly to study point sources, especially in gamma-ray astronomy, its reliability for dipole anisotropy studies with the PAMELA calorimeter had to be verified. A data subset from the experimental data was used to construct the dipole anisotropy, which looks as expected from previous measurements, but with an amplitude of order $10 \%$, see Fig. 1. The shuffling method was applied to this handmade dipole anisotropy. As it is seen in Fig. 2, the features of the simulated anisotropy disappeared, and the obtained distribution looks similar to the isotropic case (they are different because the distribution with the dipole is part of the experimental distribution). The results of this test proves that the method can be used with the PAMELA calorimeter experimental data for the dipole anisotropy search.

\section{The results}

In Fig. 3, the obtained dipole anisotropy is shown. The dipole is obtained by integrating events within $180^{\circ}$ (right accession) with shift of $5^{\circ}$ of each bin. The data set covers the time period 2006-2014. The anisotropy is measured in the equatorial coordinate system in terms of relative intensity (Karelin, 2015). The phase is $27 \pm 8$, and the amplitude is $0.0011 \pm 0.0001$. The amplitude is in excellent agreement 


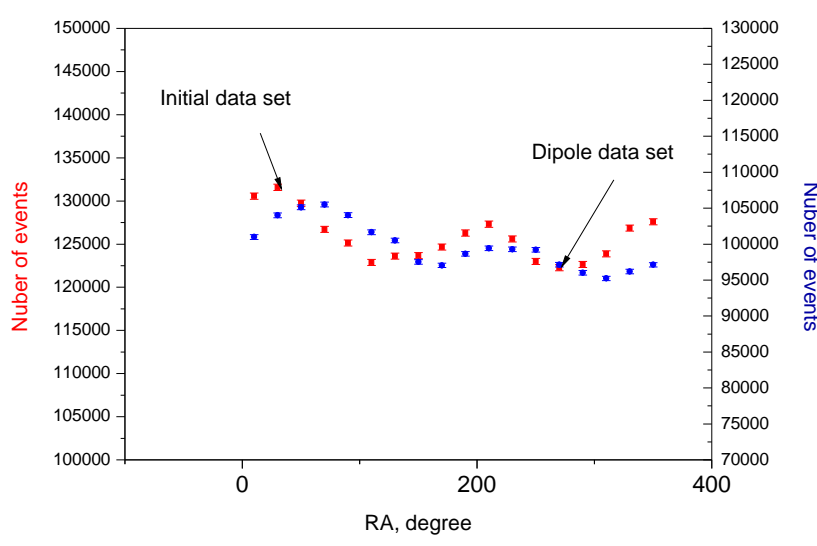

Figure 2. The comparison of the two data sets after applying the shuffling method, red dots - the initial data set and blue dots - the data with the dipole anisotropy.

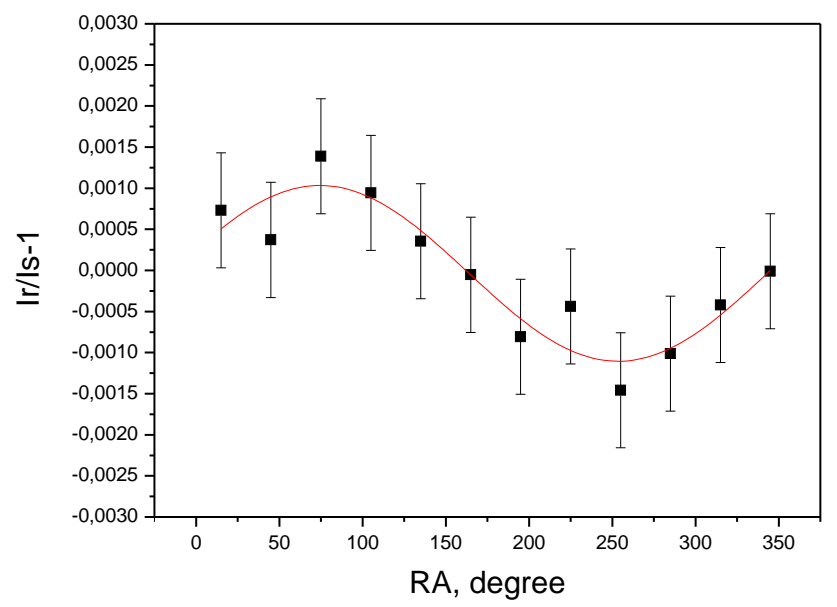

Figure 3. The Ir / Is-1 depending on RA. Ir - the real intensity, Is the simulated isotropic intensity. The red line is a fit by a sine wave.

with HAWC (BenZvi, 2014), Ice-Cube (Santander, 2013) and Bacsan results (Lidvansky et al., 2007), while the phase is also in agreement with Bacsan one and Super-Kamiokande (Guillian et al., 2007).

Acknowledgements. We acknowledge support from The Italian Space Agency (ASI), Deutsches Zentrum fur Luftund Raumfahrt (DLR), The Swedish National Space Board, The Swedish Research Council, The Russian Space Agency (Roscosmos). This work has been done with support of the Russian Science Foundation grant no. 14-12-00373 and the RF President grant MK-4599.2014.2.

Edited by: K. Scherer

Reviewed by: D. Strauss and H. Fichtner

\section{References}

Adriani, O., Barbarino, G. C., Bazilevskaya, G. A., et al.: The PAMELA Mission, Heralding a new era in precision cosmic ray physics, Phys. Rep., 544, 323-370, doi:10.1016/j.physrep.2014.06.003, 2014.

BenZvi, S.: Observations of the anisotropy of cosmic rays at $\mathrm{TeV}-$ PeV, ASTRA Proc., 1, 33-37, doi:10.5194/ap-1-33-2014, 2014.

Borisov, S. V., Voronov, S. A., Galper, A. M., and Karelin, A. V.: Reconstructing the particle direction in an extended aperture of the PAMELA apparatus using the coordinate-sensitive calorimeter, Instrum. Exp. Tech., 56, 1-8, doi:10.1134/S0020441213010053, 2013.

Guillian, G., Hosaka, J., Ishihara, K., et al. (Super-Kamiokande Collaboration): Observation of the anisotropy of $10 \mathrm{TeV}$ primary cosmic ray nuclei flux with the Super-Kamiokande-I detector, Phys. Rev. D, 75, 062003, doi:10.1103/PhysRevD.75.062003, 2007.

Karelin, A. V.: The large-scale anisotropy in the PAMELA experiment, in: Proceedings, 34th International Cosmic Ray Conference (ICRC), 30 July-6 August 2015, Hague, Holland, 2015.

Karelin, A. V., Adriani, O., Barbarino, G. C., et al.: A search algorithm for finding Cosmic-Ray anisotropy with the PAMELA calorimeter, J. Phys. Conf. Ser., 409, 012029, doi:10.1088/17426596/409/1/012029, 2013.

Lidvansky, A. S., Andreyev, Yu. M., Dzhappuev, D. D., Kozyarivsky, V. A., Kudzhaev, A. U., and Tulupova, T. I.: Direction and Magnitude of the Anisotropy of Cosmic Rays of TeV Energies, Proceedings, 30th International Cosmic Ray Conference (ICRC 2007), 3-11 July 2007, Merida, Yucatan, Mexico, 613616, 2007.

Santander, M.: Update on cosmic-ray anisotropy studies with IceCube, 33rd International Cosmic Ray Conference (ICRC2013), 2-9 July, 2013, Rio de Janeiro, Brazil, 2013. 